\title{
Las razones del miedo: deserción temprana de Beca 18
}

\section{Carlos E. Aramburú}

Antropólogo, Pontificia Universidad Católica del Perú caramburu@pucp.pe

\section{Diego Núñez}

Sociólogo, Pontificia Universidad Católica del Perú diego92.nc@gmail.com

\section{RESUMEN}

El estudio indaga sobre las razones que llevan a estudiantes de bajos ingresos, becarios del programa Beca 18, a desertar antes de iniciar los estudios o durante el primer año de los mismos. Se utilizan dos métodos: un modelo cuantitativo para comparar características familiares e individuales de desertores versus no desertores y otro cualitativo basado en entrevistas a profundidad a desertores para indagar sobre las razones de la no aceptación de la beca o retiro temprano. En este artículo se prioriza el enfoque cualitativo pues el cuantitativo no arroja diferencias significativas entre ambos grupos. La evidencia señala que los principales motivos que explican la deserción temprana o la no aceptación de Beca 18 son los factores afectivos y la falta de integración social al nuevo medio.

Palabras clave: deserción temprana, educación superior, Beca 18. 


\section{Fear as a barrier: early desertion from a public scholarship program in Peru «Beca 18»}

\section{ABSTRACT}

This article explores the reasons why low-income students, who are supported by a public scholarship program called "Beca 18", reject this support or leave their studies during the first year of studies. Results are based on two methods: a quantitative model that compares personal and family characteristics among deserters and non-deserters, and a qualitative approach based on in-depth interviews with deserters in order to inquire about the reasons for not accepting the scholarship or early withdrawal. This article prioritizes the qualitative approach because the quantitative does not show significant differences between the two groups.

The evidence indicates that the main reasons that explain the early drop-out or non-acceptance of Beca 18 are the affective factors and the lack of social integration into the new environment.

Keywords: early desertion, higher education, scholarship program, Beca 18. 


\section{ANTECEDENTES}

El Programa Nacional de Becas y Crédito Educativo (Pronabec) es la entidad oficial del Ministerio de Educación del Perú encargada de proporcionar becas $\mathrm{y}$ crédito educativo a personas con talento provenientes de sectores vulnerables y en pobreza. Pronabec surge luego de la creación del Programa Nacional Beca 18, en noviembre de 2011, como entidad para su gestión y la de otros programas similares. Posteriormente, el 12 de febrero de 2012 el Congreso de la República aprobó mediante ley 29837 la creación del Pronabec.

Pese a ser relativamente nuevo, el programa ha tenido un desarrollo exponencial. Pronabec ha otorgado en corto tiempo más becas que el Estado peruano en cuarenta años. Hasta la convocatoria de 2014 se habían otorgado 31000 becas en todas sus modalidades. El programa ha atacado centralmente el problema del acceso a la educación superior de calidad para los estratos pobres y extremadamente pobres.

Sin embargo, se vienen dando casos de jóvenes que, luego de haber culminado el proceso de selección con éxito, deciden rechazar la beca antes de su otorgamiento, renunciar a ella o perderla por diversas razones durante el inicio de la vida universitaria. El propósito de este breve trabajo es identificar las características, causas y consecuencias de esta deserción temprana de Beca 18.

\section{PROPÓSITO}

Este estudio ${ }^{1}$ tiene como objetivo recoger y sistematizar la experiencia de becarios de Beca 18 durante las etapas de aceptación de la beca y en los inicios del

Este artículo se basa en una consultoría realizada por los autores para Pronabec entre febrero y abril de 2014. Agradecemos a Jefferson José Martínez (economista) investigador asociado y la colaboración de Rosario Arias en el análisis y la de Angélica Damonte en las transcripciones de las entrevistas. 
ciclo de estudios (matrícula, inducción, recepción de clases y tutoría). Se trata de indagar sobre las diferencias entre los jóvenes que continúan sus estudios (continuadores) con los que deciden no hacerlo o no pueden hacerlo (desertores), ya sea en momentos previos a la aceptación de la beca o durante el primer ciclo de estudios superiores. Se exploran, asimismo las razones que dan estos últimos para renunciar a la beca o no continuar con sus estudios. El objetivo de política es minimizar los riesgos de deserción temprana atendiendo a las razones que, desde el propio involucrado, determinan esta decisión, contribuyendo así a una mayor eficacia y eficiencia de Beca 18 durante las etapas iniciales del proceso $^{2}$.

\section{ENFOQUE TEÓRICO Y METODOLOGÍA}

En la década de 1960 y 1970, cuando se inician los trabajos sistemáticos sobre la deserción de estudiantes universitarios, primaron las explicaciones sicológicas, es decir factores asociados al individuo en cuanto a motivación, empeño, expectativa, etc., para explicar la interrupción de los estudios superiores (Voigt y Hundrieser, 2008). Posteriormente, los trabajos de Vincent Tinto desde 1975 incorporaron las dimensiones institucionales asociadas a la retención y presentaron un modelo para explicar este hecho. La relevancia del tema radica en que, según este mismo autor, el $40 \%$ de los estudiantes universitarios de Estados Unidos no llega a graduarse y el 57\% de las deserciones ocurre en las fases iniciales del proceso educativo, antes de iniciarse el segundo año de estudios (Tinto, 1993). Esta comprobación es refrendada por trabajos en nuestra región, que encuentran que el primer año de estudios es crítico para el nuevo estudiante por la ruptura pedagógica, cultural y generacional con respecto a la secundaria (Cinda, 2006; Silva, 2011). La literatura distingue entre la dimensión de la persistencia (atribuible a los factores individuales y del contexto familiar) y la de la retención (atribuible a factores institucionales del centro de estudios: orientación, acompañamiento, mecanismos de inserción social, etc.) (Torres, 2012; Núñez, 2018). Por ello en este breve estudio nos hemos centrado en la deserción temprana (antes de, o durante el primer año de estudios), priorizando el análisis de la persistencia (más bien la falta de ella), dadas las características del programa Beca 18. Para ello, hemos analizado las razones del rechazo de la beca, es decir los motivos que aducen o

2 Queremos agradecer a la economista Kelly Cassia y al doctor Juan Arroyo, funcionarios de Beca 18, por su valioso apoyo en acceder a la información y orientaciones que han hecho posible la realización de este estudio. 
problemas que enfrentan aquellos beneficiarios que deciden no aceptar la beca antes de iniciar sus estudios o en el primer ciclo.

En el primer caso (el más frecuente), no se puede hablar de deserción de la vida universitaria, sino más bien de deserción del programa o rechazo a la beca. Ello añade una dimensión poco explorada del acceso y persistencia en la educación terciaria, incidiendo en los aspectos motivacionales y actitudinales de aquellos jóvenes que, pese a haber logrado el apoyo económico que brinda Beca 18, deciden no aceptarla por razones que presentaremos más adelante. En efecto, buena parte de la literatura internacional y nacional ha enfatizado lo económico como la barrera principal para acceder a los estudios superiores (CINDA, 2006; Casillas, Chaín y Jácome, 2007; Miller, 2009; Casillas, De Garay, Vergara y Puebla, 2001; Silva, 2011); sin embargo las investigaciones en nuestro medio se han centrado en estimar el peso de esta barrera tanto en el acceso a la educación escolar (Cueto, Miranda y Vásquez, 2000; Lavado y Gallegos, 2005), como a la superior (McLauchlan y Gallegos, 2000). Por lo tanto, esta investigación pretende ser un modesto aporte en la identificación de otras barreras más allá de la económica (aunque, como veremos, esta involucra costos adicionales al costo de los estudios), que tienen que ver con la distancia cultural entre el contexto de origen del becario y el del lugar de estudio y con las barreras afectivas.

Los trabajos académicos sobre deserción en la educación universitaria tienen como referencia central el trabajo pionero de V. Tinto, quien en 1975 presentó un modelo para explicar este hecho. La relevancia del tema radica en que, como hemos señalado, según este autor una proporción significativa de los estudiantes universitarios de Estados Unidos no llega a graduarse y más de la mitad de las deserciones ocurre en las fases iniciales del proceso educativo: antes de empezar el segundo año de estudios (Tinto, 2000). En este trabajo abordamos una dimensión poco explorada del proceso educativo, incidiendo en los aspectos motivacionales y actitudinales de aquellos jóvenes que, pese a haber logrado el apoyo económico que brinda Beca 18, deciden no aceptarla por diferentes razones.

El modelo teórico propuesto por Tinto enfatiza los procesos de adaptación e integración a la vida universitaria, incidiendo en tres de ellos que operan con cierta autonomía: el rendimiento académico, la identificación institucional que incluye la integración con los profesores y con redes sociales de amistad y compañerismo y los aspectos motivacionales. La probabilidad de desertar es, por tanto, mayor en casos de bajo rendimiento académico, pero también - y sobre todo- en los casos de falta de integración al sistema social e institucional universitario (Núñez, 2018) y en los de baja motivación para culminar los estudios. 
El modelo sugiere que los estudiantes ingresan a la universidad con los atributos familiares y propios como individuo, pero tan pronto como el estudiante es admitido, una serie de factores del sistema social lo afectan y los maestros y el grupo de compañeros lideran su desarrollo intelectual; el modelo de Tinto está basado en el involucramiento de los estudiantes donde las metas y el compromiso organizacional son los valores raíz y son facilitados por los académicos y la integración social modelo (Balmori, 2010, p. 3).

Dado el carácter exploratorio de este estudio, en el que se analiza solo la deserción temprana (antes de culminar el primer ciclo de estudios), nos centraremos en los aspectos motivacionales y las barreras «objetivas» mencionadas tanto por aquellos que no aceptaron la beca como por los que, habiendo iniciado sus estudios, no quisieron o no pudieron continuarlos.

De acuerdo con la disponibilidad de la base de datos proporcionada por Pronabec, se han utilizado dos aproximaciones metodológicas:

a) Exploración cuantitativa: en la que se comparan variables individuales, del hogar y de las instituciones educativas de origen y destino del grupo «desertor» con las de los «continuadores» de la convocatoria 2014. Ello permitió identificar las variables discriminantes entre estos dos grupos. En esta etapa se aplicó un modelo multivariable (modelo Probit), comparando estas variables relevantes entre ambos grupos para determinar los factores que inciden en la probabilidad de desertar o no. Los resultados han servido para estructurar el estudio cualitativo mediante entrevistas estandarizadas a una muestra de desertores.

b) Estudio cualitativo: basado en entrevistas en profundidad a jóvenes que no quisieron o no pudieron continuar sus estudios («desertores»). Se ha trabajado con una muestra al azar de los casos desertores ( 34 casos) de la convocatoria de 2014, a la que se aplicó una entrevista en profundidad que permitió recoger el discurso, la racionalidad y los factores que, según los propios involucrados, llevan a la decisión de no aceptar la beca o no poder o querer continuar con sus estudios durante el primer año de estudios. Las entrevistas, para las que se contó con autorización explícita de los involucrados, se realizaron por medio telefónico; ello permitió grabar los testimonios y respetar la privacidad garantizando la confidencialidad.

La utilización de dos métodos complementarios (cuantitativo y cualitativo) confiere robustez y mayor objetividad al estudio. Permite, además, abordar 
en mayor detalle los factores «objetivos» (exógenos al discurso del becario) y «subjetivos» (endógenos al discurso del becario) para entender mejor las causas y razones detrás de la deserción temprana.

\section{RESULTADOS}

En la convocatoria de 2014, hubo 655 casos de deserción ${ }^{3}$ de un total de 13369 beneficiarios seleccionados por PRONABEC para acceder a una beca integral de estudios superiores en las Instituciones de Educación Superior (IES) y para las carreras elegibles. Este número de casos representa cerca de un $5 \%$ de deserción, muy por debajo de las tasas observadas en América Latina para estudiantes de educación terciaria en general: 57\% (Cinda, 2006, tabla 3, p. 31). Dada esa situación, el presente estudio tiene como objetivo conocer con mayor profundidad y detalle los factores que afectan la permanencia de becarios de grupos vulnerables con alto rendimiento escolar que acceden a Beca 18 y desertan antes de comenzar o durante el inicio de los estudios superiores en las IES elegidas.

\section{Modelo cuantitativo}

Para el análisis cuantitativo se trabajó con variantes de un modelo Probit, considerando todos casos de la convocatoria 2014. Para ello, se planteó un modelo de análisis a partir de trece variables individuales, educativas, económicas y familiares. El objetivo fue encontrar su relevancia para la probabilidad de desertar.

Las hipótesis tentativas que buscamos probar con estos modelos eran que, respecto de las variables individuales, las mujeres tendrían mayor probabilidad de desertar que los varones, debido a rasgos culturales de mayor protección hacia las hijas, especialmente si la IES elegida quedaba lejos de su hogar; que los becarios de mayor edad tendrían mayor probabilidad de desertar por presiones laborales y familiares y que los jóvenes que trabajaban tendrían mayor probabilidad de desertar por la pérdida de ingresos económicos. En cuanto a los factores educativos-institucionales, hipotetizábamos que los que estudiaban en IES privadas tendrían más probabilidad de desertar por la mayor exigencia en los estudios y que los becarios provenientes de colegios públicos tendrían mayor probabilidad de desertar que los de colegios privados por la menor calidad de la educación recibida en los primeros.

\footnotetext{
3 Incluye becarios a los que se adjudicó beca, pero nunca se matricularon.
} 
En cuanto al origen socioeconómico, asumíamos que los becarios provenientes de hogares más humildes (usando como proxy las características de la vivienda: tipo de piso y acceso a luz), tendrían mayor probabilidad de desertar por no contar con recursos mínimos para gastos asociados a la beca. Finalmente, el modelo explora el peso de las variables familiares, sosteniendo que los becarios procedentes de hogares con padres de mayor edad y con bajo nivel educativo tendrían mayor probabilidad de desertar por recibir un menor apoyo para continuar sus estudios y por depender estos padres en mayor medida de la contribución económica de sus hijos; de igual manera habría un mayor riesgo de deserción en los casos en que la madre trabajara, por ser el becario un apoyo económico clave para la familia.

Respecto de los resultados del modelo cuantitativo, estos tuvieron una capacidad explicativa limitada dado que las variables independientes no fueron significativas. Por lo tanto, fue necesario utilizar alguna variación al modelo teórico propuesto con el fin de identificar las variables que ayudaran a explicar «mejor» la probabilidad de un becario de desertar o continuar los estudios. Por ello se trabajó modelos individuales para cada tipo de variable con el objetivo de identificar las que tuvieran mayor capacidad explicativa sobre la deserción.

A partir de los resultados de los modelos ajustados, las variables con mayor fuerza explicativa fueron: edad del becario, tipo de IES elegida (privada o pública), piso de la vivienda, tipo de alumbrado en la vivienda, grado de instrucción del padre, grado de instrucción de la madre y si la madre trabaja. Por lo tanto, el paso siguiente fue estimar la probabilidad de los becarios de desertar o no, a través de un modelo que solo tomara en cuenta este grupo de variables y analizar si todas eran significativas.

Tras estimar el modelo ajustado, se obtuvo como resultado que casi todas las variables eran significativas al 5\%, excepto las educativas-institucionales. Por tal motivo, se procedió a estimar un modelo similar, pero que no incluyera dichas variables. Con este último modelo encontramos que ninguna de las variables analizadas generaba un cambio mayor a $1 \%$ sobre la probabilidad de desertar o no al programa. De esa manera, se evidencia que los modelos estadísticos no brindan evidencia concluyente sobre las diferencias entre los becarios que desertan al programa y los que no lo hacen. En otras palabras, a pesar de ser las más significativas, ni las características individuales (mayor edad), la condición económica (carecer de electricidad en el hogar) ni las características de los padres (menor nivel educativo y trabajo de la madre) tienen la suficiente relevancia, en términos estadísticos, para explicar las diferencias entre los desertores y los continuadores. 
El análisis estadístico nos lleva a dos conclusiones. En primer lugar, la población de becarios es muy homogénea en cuanto a estas características «gruesas», por lo que no se pueden encontrar diferencias significativas como probablemente ocurriría si comparamos la deserción entre becarios y no becarios. En segundo lugar, se refuerza la necesidad de indagar sobre variables más «finas» referidas al proceso de toma de decisiones y el peso de variables culturales, actitudinales y del mismo proceso de obtención de la beca e inicio de los estudios. Precisamente para abordar estos aspectos se aplicó la metodología cualitativa que se describe a continuación.

\section{RESULTADOS DEL ESTUDIO CUALITATIVO}

\section{Metodología}

Para la parte cualitativa del estudio, se trabajó con una muestra al azar de desertores de la convocatoria 2014, con la finalidad de aplicar entrevistas a profundidad que permitieran conocer el discurso, la racionalidad y los factores que, según los involucrados, los llevaron a tomar la decisión de no comenzar los estudios o desertar en el primer ciclo de estudios. Dado que la unidad de análisis fueron los jóvenes que renunciaron a Beca 18 y que residen en el Perú, se decidió acceder a ellos mediante entrevistas telefónicas vía celular. Esto permitió tener un diálogo más fluido que si hubiéramos utilizado entrevistas presenciales, por correo o e-mail. Para la entrevista telefónica se elaboró una guía que tomaba en consideración tanto dimensiones «objetivas» (hechos), como «subjetivas» (valoraciones, sentimientos, actitudes) sobre cuatro dimensiones: variables individuales, familiares, de obtención de la beca y de la deserción.

Se decidió entrevistar como mínimo a treinta jóvenes como informantes anónimos y confidenciales ${ }^{4}$. Para ello se realizó una selección al azar simple de cincuenta casos (treinta entrevistables y veinte reemplazos) entre los 655 desertores existentes. En el proceso de entrevistas surgieron algunas dificultades: 76 celulares de los desertores seleccionados estaban apagados, diecisiete de los números de contacto eran equivocados, en once casos no contestaron las llamadas, hubo once celulares que tenían la línea fuera de servicio, en dos casos hubo pro-

4 Tratándose de un estudio cualitativo el tamaño muestral $34 / 665=>5 \%$ es irrelevante, lo clave es que la selección sea al azar para asegurar la representatividad de los informantes. No importa a cuántos se entrevista, sino a quién se entrevista. 
blemas de comunicación, y en dos casos más, no se encontraban pues el número era de un familiar. El siguiente cuadro resume el rendimiento de la muestra:

Tabla 1. Rendimiento de las muestras de desertores Beca 18 para entrevistas telefónicas(2014)

\begin{tabular}{lc}
\hline Celular apagado & 76 \\
Aceptó entrevista & 34 \\
Rechazó entrevista* $^{*}$ & 22 \\
Número equivocado & 17 \\
No contestó & 11 \\
Línea fuera de servicio & 11 \\
Problemas con la comunicación & 2 \\
No se encontraba/número de familiar & 2 \\
\hline Total & 175 \\
\hline * De los veintidós que rechazaron la entrevista, diecisiete son retiros volun- \\
tarios, cuatro reprobaron y uno sin información.
\end{tabular}

Otra de las dificultades para poder acceder a los informantes fue el tiempo. Había que realizar las entrevistas los fines de semana o los días de semana en las tardes y noches, pues estos jóvenes trabajan o estudian, por lo que en otros días y horarios no era posible que accedieran a ser entrevistados. Cabe mencionar que también hubo veintidós casos en que los entrevistados no quisieron ser entrevistados o grabados.

Como se observa en la tabla 1, se logró registrar 34 entrevistas en total. Para conseguir tal número se tuvieron que realizar tres muestreos al azar, dos de cincuenta casos y el último de treinta. El propósito de estos tres muestreos fue asegurar un número suficiente de casos; el hecho de que en todos estos se mantuviera la selección aleatoria (por la que todas las unidades muestrales del universo, en este caso los desertores tienen igual probabilidad de selección: 1/655) asegura la confiabilidad de los resultados. Todos los entrevistados eran menores de veinticinco años por ser más representativos. Los casos que no cumplían con esta condición (cinco) se encontraban exclusivamente en las modalidades Beca 18 Fuerzas Armadas y Beca 18 Especial Reparaciones en Educación. En general, la edad de los entrevistados fluctúa entre los dieciséis y los veintitrés años, con 
excepción del entrevistado de veintiocho años de la modalidad Fuerzas Armadas. En promedio, la edad de los entrevistados es de 18,85 años.

En total tomó dos semanas realizar las 34 entrevistas. En promedio las entrevistas tuvieron una duración de quince minutos con quince segundos, siendo la más corta de nueve minutos con cuatro segundos y la más larga de veinticinco minutos con 48 segundos. Una dificultad adicional a las ya explicadas fue el problema de comunicación telefónica con provincias, lugar de residencia de una buena parte de los casos seleccionados. Debido a esto, algunas entrevistas no se pudieron realizar por mala comunicación; en otros casos se cortó la llamada y se grabó la entrevista por partes.

\section{Caracterización de los entrevistados}

En esta sección analizaremos quiénes son los desertores entrevistados en función de sus rasgos «objetivos» (edad, sexo, características familiares, lugar de origen, etc.) que, como se demuestra en el estudio cuantitativo, no difieren significativamente de aquellos de los «continuadores», para luego presentar y discutir las dimensiones «subjetivas» asociadas a la deserción.

Con relación al sexo de los entrevistados, se logró una distribución equitativa en esta muestra. Se entrevistó a dieciocho hombres y dieciséis mujeres (tabla 2).

Tabla 2. Distribución por sexo y edad de los entrevistados

\begin{tabular}{lllllllllllll}
\hline & \multicolumn{10}{c}{ EDAD } & \multirow{1}{*}{ TOTAL } \\
\cline { 2 - 11 } Sexo & 16 & 17 & 18 & 19 & 20 & 21 & 22 & 23 & & 28 & \\
\hline Masculino & -- & 2 & 6 & 3 & 2 & 1 & 1 & 2 & -- & -- & 1 & 18 \\
Femenino & 1 & 6 & 3 & 4 & 2 & -- & -- & -- & -- & -- & -- & 16 \\
TOTAL & 1 & 8 & 9 & 7 & 4 & 1 & 1 & 2 & -- & -- & 1 & 34 \\
\hline
\end{tabular}

Excluyendo al entrevistado de veintiocho años, la edad promedio de los varones es 18,2 años y la de las mujeres 18 . En cuanto al contexto familiar y posición de los entrevistados dentro de su familia, la muestra presenta las siguientes características:

En lo relativo al tamaño familiar más de la mitad son parte de familias numerosas (más de cuatro hijos) rasgo propio de hogares rurales pobres. La mayoría son hijos mayores o intermedios. 
Tabla 3. Sexo y posición dentro de la familia

\begin{tabular}{lccccc}
\hline & \multicolumn{5}{c}{ Posición en la familia } \\
\cline { 2 - 6 } Sexo & Mayor & Intermedio & Menor & Hijo único & No dice \\
\hline Masculino & 6 & 6 & 5 & 1 & \\
Femenino & 5 & 6 & 3 & 1 & 1 \\
Total & 11 & 12 & 8 & 2 & 1 \\
\hline
\end{tabular}

Tabla 4. Tamaño de la familia $\left(\mathrm{N}^{\circ}\right.$ de hermanos)

\begin{tabular}{lc}
\hline Hijo único & 2 \\
Dos a tres hijos & 12 \\
Cuatro a cinco hijos & 10 \\
Seis a más & 8 \\
No contesta & 2 \\
\hline Total & 34 \\
\hline
\end{tabular}

Hay menos mujeres menores que varones y muy pocos hijos únicos. Ello se asocia a familias numerosas, que evidencian estar en una fase previa al descenso de la fecundidad; en efecto, en la tabla 4 se aprecia que más de la mitad de las familias de los entrevistados (dieciocho) tiene más de cuatro hijos, y ocho familias seis o más hijos. Una familia numerosa es más frecuente en el medio rural; así, diecinueve de los 34 entrevistados provienen del medio rural (aldeas o comunidades campesinas) y tienen a sus padres ocupados en la agricultura y ganadería familiar.

La mayoría de los entrevistados (veintiuno) vivían con sus padres y hermanos al momento de postular a Beca 18 y siete vivían solo con la madre por fallecimiento o abandono del padre, lo que revela mayor vulnerabilidad. Tres ya vivían solos y uno con su abuela.

Tabla 5. Nivel educativo de los padres

\begin{tabular}{lcccccc}
\hline & $\begin{array}{c}\text { Sin } \\
\text { instrucción }\end{array}$ & $\begin{array}{c}\text { Primaria } \\
\text { incompleta }\end{array}$ & $\begin{array}{c}\text { Primaria } \\
\text { completa }\end{array}$ & $\begin{array}{c}\text { Secundaria } \\
\text { incompleta }\end{array}$ & $\begin{array}{c}\text { Secundaria } \\
\text { completa }\end{array}$ & Superior \\
\hline Padre & 1 & 1 & 7 & 6 & 8 & 2 \\
Madre & 3 & - & 12 & 8 & 5 & 1 \\
Total & 4 & 1 & 19 & 14 & 13 & 3 \\
\hline
\end{tabular}


Los entrevistados pertenecen a familias cuyos padres tienen bajos niveles educativos: diecinueve de los progenitores solo alcanzan la primaria; catorce tienen secundaria incompleta, y trece, secundaria completa. Solo tres de los padres (dos padres y una madre) cuentan con estudios superiores, mientras que de los cuatro que carecen de instrucción formal, tres eran mujeres. Como ocurre en los estratos más pobres, las madres tienen menores niveles educativos que los padres; veinticuatro madres tienen solo primaria o menos (tabla 5).

Consistente con lo encontrado en el punto anterior, la mayoría de los progenitores trabaja en sus parcelas familiares dedicándose a la agricultura y ganadería (33), mientras que seis se dedican al comercio en pequeña escala. Son escasos los trabajadores calificados u obreros. Todo ello revela el bajo estatus socioeconómico de la familia de origen de los desertores.

La región de procedencia de los entrevistados es bastante diversa, lo que revela una amplia distribución geográfica de Beca 18 y una buena representatividad territorial de nuestra muestra. La mayoría de los entrevistados proviene de la zona andina.

Sobre la ubicación del centro de estudios elegido por los entrevistados, encontramos que en quince de los 34 casos eligieron una IES ubicada en Lima, pese a que ninguno de ellos habitaba en la capital (tabla 6).

Tabla 6. Ubicación del IES elegido por el becario

\begin{tabular}{lc}
\hline Región & $\mathrm{N}^{\mathrm{o}}$ \\
\hline Lima & 15 \\
Junín & 4 \\
Ayacucho & 2 \\
La Libertad & 2 \\
Lambayeque & 2 \\
Piura & 2 \\
San Martín & 2 \\
Apurímac & 1 \\
Huancavelica & 1 \\
Huánuco & 1 \\
Puno & 1 \\
Tacna & 1 \\
Total & 34 \\
\hline
\end{tabular}


Si bien ello revela una preferencia por las IES ubicadas en la capital ${ }^{5}$, presumiblemente por su mayor prestigio y calidad, como veremos más adelante la lejanía es una razón para la deserción debido a la falta de contacto con el hogar y carencias afectivas.

Con respecto al tipo de IES elegido, diecinueve de los entrevistados ingresaron a universidades y quince a institutos de educación técnica. Encontramos una marcada preferencia por instituciones privadas (32 de los 34 casos) por asociarlas a mayor prestigio y calidad de la enseñanza. En cuanto a las carreras elegidas, veinte ingresaron a diversas ramas de la ingeniería, cinco a carreras relacionadas con la educación, otros cinco a especialidades de informática y el resto a carreras administrativas o afines. Esto demuestra que existe una amplia diversidad en cuanto a las carreras seleccionadas y confirma la inexistencia de sesgos de selección con relación a nuestra muestra. Cabe notar que el programa Beca 18 establece las especialidades para las que se puede postular a dicha beca.

\section{La deserción: hechos y razones}

A continuación se abordará la evidencia referida al proceso de deserción, enfocándonos tanto en sus características como en las razones presentes en el discurso de los entrevistados.

En la tabla 7 se registra la primera razón para la deserción con la que las y los entrevistados explican su decisión. En la primera parte de esta tabla se recogen las razones de los desertores que no llegaron a comenzar sus estudios, mientras que en la segunda las de aquellos que sí lo hicieron.

Es importante resaltar que el cuadro anterior ha sido realizado tras un esfuerzo por identificar una razón principal, pero en la mayoría de casos, los entrevistados mencionan varias razones que se entrecruzan, por lo que es difícil atribuir preponderancia a una sola. En el análisis es importante distinguir el momento en el que los entrevistados renunciaron a la beca, por lo que la tabla 7 diferencia las razones según el momento de la deserción (veintitrés de los desertores no comenzaron sus estudios, frente a once que sí lo hicieron). Como veremos más adelante, los motivos de la deserción son complejos e involucran muchas dimensiones tanto subjetivas como objetivas.

\footnotetext{
Tener en cuenta que la mayor oferta de IES para Beca 18 proviene de Lima.
} 
Tabla 7. Razón de deserción identificada como principal por el entrevistado según sexo

\begin{tabular}{lccc}
\hline Desertores que no iniciaron los estudios & & & \\
\hline Razón & $\mathrm{N}^{\mathrm{o}}$ & Hombres & Mujeres \\
\hline Problemas económicos & 6 & 3 & 3 \\
Enfermó & 3 & 3 & 0 \\
Embarazo de su pareja & 2 & 2 & 0 \\
Enfermedad de familiar & 2 & 2 & 0 \\
Infringió un requisito (inició estudios en otra IES) & 2 & 2 & 0 \\
Problemas viviendo solo o sola & 2 & 0 & 2 \\
Iba a extrañar a su familia & 1 & 0 & 1 \\
Miedo a fracasar académicamente & 1 & 1 & 0 \\
Problemas con los documentos & 1 & 1 & 0 \\
Problemas vocacionales & 1 & 0 & 1 \\
Su familia no lo dejó vivir solo & 1 & 1 & 0 \\
\hline Subtotal & 22 & 15 & 7 \\
\hline Desertores que sí iniciaron los estudios & & & \\
\hline Razón & $\mathrm{N}^{\mathrm{o}}$ & Hombres & Mujeres \\
\hline Problemas vocacionales & 3 & 1 & 2 \\
Problemas académicos & 3 & 1 & 2 \\
Extrañaba a su familia & 2 & 0 & 2 \\
Problemas económicos & 2 & 2 & 0 \\
Enfermedad de familiar & 1 & 1 & 0 \\
Problemas viviendo solo(a) & 1 & 1 & 0 \\
\hline Subtotal & 12 & 6 & 6 \\
\hline Total & 34 & 21 & 13 \\
\hline
\end{tabular}

Aunque nuestra muestra no tiene pretensiones de representación estadística, es revelador que, según este resultado, dos de cada tres desertores no llegan a iniciar sus estudios, lo que indica que las barreras o dificultades se presentan con mayor peso en la etapa previa a los estudios. Volveremos sobre este punto más adelante. 
Al presentar las razones de deserción de los entrevistados ${ }^{6}$, es importante indicar que el discurso revela una asociación compleja entre varias razones, en especial en el caso de las jóvenes, por lo que resulta difícil determinar una única razón detrás de su decisión. Más bien lo que encontramos son razones asociadas, especialmente una combinación de persistencia de barreras económicas (CINDA, 2006) con razones afectivas y de inseguridad ante la perspectiva de residir solos en una ciudad desconocida. Pese a ello, hemos intentado clasificar las razones encontradas en el discurso en temas referidos a barreras afectivas e inseguridad, barreras económicas, problemas de salud tanto familiares como del propio becario, problemas vocacionales y de bajo rendimiento (para los que inician sus estudios) y problemas asociados al mismo proceso y a las normativas del programa.

\section{Las razones del miedo}

Como se ha mencionado, la mayoría de los entrevistados proviene de pueblos o aldeas del interior y de familias rurales y numerosas con fuertes nexos afectivos y funcionales. Además, la mayoría de jóvenes entrevistados trabaja con sus padres y familiares en el negocio familiar (habitualmente la chacra). En su discurso se aprecia una notable fuerza e importancia de los lazos familiares. Por ello es que la lejanía geográfica y cultural del lugar de estudios, Lima en el mayor número de casos, dificulta la decisión de dejar su entorno familiar y local para aventurarse a la gran ciudad. Esta es percibida como «extensa», «cara», «difícil»y «peligrosa». Por ello no debe sorprender que, en cerca de la mitad de los entrevistados, la barrera principal para iniciar o continuar los estudios superiores, especialmente para las jóvenes, sea la afectiva y emocional como es evidente del análisis del discurso:

[Mis familiares] me estaban diciendo: «¿Cómo vas a Lima? Los cuartos son tan caros, no sé adónde vas a estar, como no hay ningún familiar [en Lima], no sé cómo vas a hacer» (FI29).

Bueno, [lo más difícil al empezar los estudios] fue alejarme de mi familia, porque estaba lejos la universidad [...] Me fui con mi papá, estuvo tres días nada más y después mi papá ya se vino y no me acostumbraba, estaba lejos y tuve que volverme (FI28).

[Tuve que dejar la beca] porque no tuve donde llegar allá en Lima, porque no tengo familiares allá en Lima (FI25).

6 Guardando la confidencialidad, se ha identificado cada cita textual por el sexo (M/F) y el número del informante (I). Los resaltados son nuestros. 
[Lo más difícil fue] el cambio más que todo y no estar ahí con la familia, estar lejos, no conocer nada, tal vez extrañar Moquegua y mi casa, eso al inicio cuando comencé con la beca (MI24).

Cuando me fui estuve los primeros meses, pero la verdad no me acostumbraba por el lugar, además que estaba sola (FI9).

Esa sensación de lejanía, de inseguridad, de miedo a estar solos lejos de la familia es compartida por los padres en muchos casos, lo que reforzaba la decisión de renunciar a Beca 18:

[La decisión de no aceptar la beca fue] en sí de los dos [mis padres y mía], porque también querían [que acepte la beca], pero lamentablemente no había esa posibilidad de ir porque no tenía adonde llegar en sí; si no, ¡cuánto me hubiera gustado estudiar ahí! (FI25).

Complicado fue, porque no solo tenía que pensar en mí, también tenía que pensar en mis padres, porque les afectaba emocionalmente porque yo jamás me habia separado de mis padres (FI22).

[Mi familia se preocupa] a veces sí por lo que yo tenía que venir acá a Lima sola, no conocía bien, no conozco bien hasta ahora y todo lo que pensaba, ¿con quién voy a quedar? ¿Y si me pierdo? (FI18).

Sí, se me complicó, porque como tenía que viajar una hora [a mi casa], tenía que alquilar cuarto y se me complicó porque mis padres no estuvieron de acuerdo que yo viviera allá solo en Chiclayo (MI10).

Me quedé en la Universidad de Tacna porque mi mamá no quería que vaya para Lima porque iba a estar sola a pesar de que me dan el cuarto y todo siempre, ¿no? (FI12).

[Mi mamá] tenía temor de que fuera a Lima, porque es otra manera de vivir diferente y por los peligros que hay en Lima [P: ¿Qué hubiera tenido que pasar para que continuaras con tus estudios?] Que mi mamá se hubiera ido a vivir allá (FI21).

Otra constante cultural encontrada en el discurso alude no solo a la cercanía afectiva, sino también al apoyo que esperan recibir de sus padres para persistir en los estudios:

[Mi mamá] siempre se va a preocupar, cuando estamos alejados $m i$ mamá siempre se preocupa por mí, siempre está pendiente, porque estar alejado de la familia siempre es complicado (vive en La Merced, estudiaría en Huancayo) (MI15). 
Nosotros como adolescentes necesitamos estar más cercanos a los padres, oportunidades de estudios más cerca, no tan alejado. [Los hijos] se dedican a otra cosa y no tanto al estudio. Los padres deben estar aconsejándoles a los hijos que deben ir adelante, no para atrás (FI19).

Entre los entrevistados, encontramos dos jóvenes de 28 y 23 años que tuvieron que renunciar a la beca ante la presión familiar para que trabajasen, pues habían embarazado a sus parejas:

No me dejaron ir, me dijeron que no, cómo me vieron que está con la chica: «¿Cómo a esa mujer la vas a dejar ahí?». Vinieron los de la mujer acá y comenzaron a hacer problemas que no: «¿Qué vas a hacer, la vas a dejar botada?». Me quisieron pegar y todo. Entonces dije que ya no se iba a poder (MI1).

La verdad es que no la pude continuar porque yo tuve un problema. Lo que pasa es que yo tenía mi enamorada y ella salió en estado. Sus padres, más que todo, llegaron a intervenir y dijeron que si yo la dejaba abandonada me iban a meter juicio (MI2).

En estos dos casos, la deserción no fue decisión del becario sino una imposición de los familiares de la pareja, e incluso de su propia familia. Nótese que en ambos casos se trata de varones que están por encima del promedio de edad de los becarios del programa.

El discurso revela la gran importancia que dan los entrevistados, en especial las jóvenes, a la cercanía afectiva y física con sus familias, sobre todo sus padres, quienes no solo constituyen un soporte emocional indispensable, sino además un factor que apoya la motivación y continuidad en los estudios. Se contraponen a ello los miedos, explicables en jóvenes que todavía son casi adolescentes, de estar solos en una ciudad y un contexto urbano que no solo les es extraño, sino que además es percibido como caro, complicado, desconocido y hostil. Por tanto, es necesario que el programa trabaje en crear nuevos lazos afectivos y de amistad entre los becarios, en especial los que asisten al mismo centro educativo y facilite la transición e integración socioemocional al nuevo contexto de vida, como sugerimos en la sección final de este breve estudio.

\section{La persistente barrera económica}

Para los sectores de bajos ingresos, la principal barrera de acceso a la educación superior es la económica (INEI, 2010a). En el quintil más pobre, solo el $10 \%$ de los jóvenes menores de veintidós años asisten a un centro univer- 
sitario frente al 35\% de los del quintil más rico. Sin embargo, en el acceso a la educación técnica casi no hay diferencias: $12 \%$ en el Q1 vs. $10 \%$ en el Q5 (INEI, 2010a). También la deserción está asociada con la necesidad de trabajar y generar ingresos personales y para apoyar a la familia. Por tanto, al cubrir Beca 18 el costo de los estudios y la manutención, esta barrera debería desaparecer. En efecto, es muy probable que la baja tasa de deserción del programa (menos del 5\% no inician o desertan en el primer año, frente al 15\% a $30 \%$ en estudiantes no becados) se explique por el apoyo económico que recibe el becario. Sin embargo, según las versiones recogidas en ocho de las entrevistas, persisten problemas económicos que llevan a los becarios a no poder iniciar sus estudios o continuarlos. Entre las barreras económicas señaladas, en especial por aquellos que no pudieron iniciar sus estudios, figura el no poder cubrir el costo de los pasajes para trasladarse al lugar de estudios:

Un percance fue la economía que no podía viajar (desde la selva a Lima) y pedían que nosotros demos el pasaje y después me lo iban a reembolsar pero no teníamos recursos económicos, no alcanzaba el dinero (MI14).

Solo me dijeron que trate de conseguir ese dinero para poder llegar a la universidad (en Lima) y no pude conseguirlo y no llegué a estudiar en la universidad (MI16).

También se menciona la imposibilidad de conseguir el dinero para el viaje y el alojamiento durante las primeras semanas de estadía en el lugar de estudio, lo que se ve agravado por la poca antelación con que se les avisa que deben viajar:

El mes de abril decía que todavía no depositaban a los estudiantes y que van a depositar dentro de quince días y ese mismo día teníamos que viajar a Lima porque las clases ya empezaban, entonces yo dije yo no tengo nada, mi papá está en el hospital y mi mama es la que trabaja y no gana nada, yo eso pensaba, ¿cómo hago? Mejor renuncio, digo así (FI18).

La precariedad económica del becario y sus familias dificulta agenciarse incluso de fondos modestos para cubrir los gastos iniciales en tanto el programa les proporciona el dinero:

Yo pedí prestado y me decían que no tienen, que qué beca va a estudiar, entonces ya no logré [reunir el dinero] me puse triste y dije mejor voy a renunciar, ya será entonces en otra oportunidad... si es que hay otra oportunidad (FI18).

No solo es el costo del viaje, sino además los gastos iniciales de instalación (alojamiento, comida y pasajes locales) que el becario debe afrontar con sus propios recursos: 
Sí, me avisaron muy tarde y aparte me dijo el coordinador que el primer mes no se iba a pagar y teníamos que hacer con nuestros propios gastos, entonces prácticamente yo no tenía familiares, nada, yo no conocía Lima, que es muy extenso... Entonces ya no me animé a ir porque ¿dónde quedaría la universidad, cómo llegaría? Nada, y nos quedamos (MI17).

Yo misma tomé la decisión, mi papá me dijo hija tú no te preocupes por mí (estaba en el hospital)...si hubiese tenido el dinero suficiente para poder sustentarme esos quince días que iban a pagar... Mi papá lloró, él pensaba que era por su culpa, no tenía ni un sol en el bolsillo y tenía que venir acá (a Lima), donde quedarme... Esa es la voluntad de Dios (FI18).

Me dijo mi prima que cuando llegas ellos no te buscan el cuarto sino que te dicen búscatelo, y como no conoces ¿de dónde vamos a conseguir para hospedarnos? Y ya estaba más de una semana ahí y no los apoyaban nada, dijeron que en agosto vamos a depositar el dinero y entonces ¿con qué dinero vamos a estar? Porque saben que el menú cuesta más allá... Entonces ya no viajé, ya (FI27).

Finalmente, encontramos la percepción de Lima como una ciudad muy cara y dudas acerca de si el estipendio de 1200 soles mensuales será suficiente para cubrir los gastos:

Me daban un presupuesto muy bajo, S/ 1200 que no me alcanzaba absolutamente para nada. Además, la facultad era en La Molina (UPCH), un lugar pituco y todo eso... y en Lima prácticamente nada es barato, todo es caro... (FI13).

Otros informantes hacen referencia a los costos de copias y de presentación de documentos que sustentan de sus gastos:

Me pedían materiales, un montón de cosas... documentos, declaración jurada, boletas de la laptop... me salí porque ya no podía presentar los documentos, no me alcanzaba el dinero para sacarlos, ni para pasajes, nada. Había que sacar copias, etc. (MI3).

[Dejé de estudiar] por motivos económicos. Claro, [la beca me apoyó económicamente] pero antes de eso para hacer los papeleos y todo lo que nos piden en la beca tuve que invertir plata y ya se me había agotado y no tenía con qué y todavía no tengo, y me retiré. (MI7).

En resumen, las barreras económicas señaladas revelan la precariedad financiera de los desertores y sus familias, que carecen de los recursos para cubrir incluso costos mínimos como pasajes, trámites y solventar los costos de aloja- 
miento, comidas y movilidad local durante las primeras semanas de estadía en el lugar de estudio. Ello se complica, pues a muchos les avisan (o se enteran) a última hora que deben viajar, en especial a Lima, dificultando que puedan lograr un préstamo o agenciarse del dinero necesario para ello. Se aprecian también razones «subjetivas» sobre el alto costo de vida, principalmente en Lima y la inseguridad de que el monto asignado por Beca 18 les alcance para vivir y estudiar en una ciudad en la que no conocen a nadie. Las barreras económicas aparecieron con mayor independencia y nitidez (no asociadas a otras razones) entre los varones y son las más mencionadas luego de las barreras afectivas.

\section{La mala salud}

En el discurso de siete de los entrevistados encontramos referencias a problemas de salud, cinco referidos a ellos mismos y dos a familiares cercanos. En todos los casos de enfermedad personal o familiar, esta ocurrió antes de iniciar los estudios. Algunos casos son dramáticos, pues revelan que pese a la firme decisión y motivación para continuar con Beca 18, la enfermedad o dolencia se los impidió:

Yo postulé el año pasado. Tenía que iniciar las clases en julio, pero yo estaba enfermo y no me presenté al SENATI. Estuve bastante tiempo internado en Chanchamayo Primero me diagnosticaron con dengue, pero los médicos no tenían el conocimiento. Tienes que irte al hospital de Lima [me dijeron], y me trasladaron por emergencia al Carrión. Luego de varios exámenes me diagnosticaron cáncer. Estoy siguiendo el tratamiento con quimioterapia. Cada veinte días tengo que ir a Lima (MI15).

Antes de que me dieran Beca 18, la laptop, yo me enfermé, me fui a Huancayo en el (hospital) Carrión estaba, dos meses en cama ... me enfermé de los pulmones $(T B C)$. Ya no aguantaba, no podía estar sentado. Estuve en tratamiento un año sin poder salir de mi casa (MI32).

Habia sufrido un accidente, toda mi columna estaba mal porque sufro escoliosis, y toda mi columna se dobló. Ya no pude conversar con los de Beca 18, estaba muy lejos (Lucanas) y lo dejé (MI23).

En otros casos, problemas de salud menos serios se combinan con otras razones que parecen ser las principales:

Estaba insegura, me dio temor quedarme sin dinero en Lima. El nivel académico de Lima es mucho más que acá en Huancavelica, por eso renuncié. Además, estaba con gripe y con tos (MI22). 
No me gustaban las dos carreras a las que postulé, y yo decía [a mi mamá] que no me sentía bien conmigo misma, yo le pedí que mejor lo dejemos ahí y salí de Beca 18 y también por enfermedad, porque me puse mal, me dio como depresión y se me complicaron varias cosas (FI9).

Finalmente, tenemos casos en que la enfermedad de un familiar cercano, combinada con otras razones, contribuyó a la decisión de abandonar Beca 18:

No logré coger la Beca 18 por motivos de que mi madre se sentía sola y ahi nomás cayó un poco mal y ya no la pude coger, cuando quise cogerla ya era tarde, ya. Ya no había opciones, ya (MI30).

Lo más difícil fue tener que dejar a mi familia. También tengo un hermano que postuló, pero no le alcanzó el puntaje. No quería dejar a mi hermano, a él no le salió tampoco, porque mi hermano iba a venir. Yo sí [también iba a viajar], pero se enfermó mi abuelita y ya no pude viajar (FI34).

Como se aprecia en estos dos casos, las jóvenes aducen la enfermedad de un familiar como razón (¿o pretexto?) adicional para no haber aceptado la beca.

En los primeros casos descritos, se constata la angustia de los jóvenes de perder la oportunidad de estudiar por razones fuera de su control y la ilusión de que sus casos puedan ser reconsiderados:

Ellos [Beca 18] habian sacado una resolución declarándome en abandono de estudios voluntario... pero en realidad no fue voluntario, fue por motivo de la enfermedad (cáncer). Se lo expliqué a ellos y se lo comuniqué a SENATI. Mi mamá ha hecho el trabajo de ir a SENATI y ver cómo podía yo hacer para reservar esa beca. Me acerqué a las oficinas de Beca 18 (en Lima) en febrero, ellos se conmovieron con mi caso y me ofrecieron su apoyo. Me dijeron tú tienes que esperar que salga una resolución que va a ser publicada en la página web, pero hasta la fecha de hoy no ha salido la resolución (MI15).

Les dije que yo no podía estudiar porque mi espalda ya no aguanto (TBC), si se puede entrar el próximo año, y me dijo, ya no se puede jovencito, si tú dejas ya no puedes, ya. Mi mamá también lloró, estaba triste porque ya no voy a poder estudiar (MI32).

Como se aprecia, es fundamental atender aquellos casos en los que la enfermedad priva temporalmente a estos jóvenes de la posibilidad de estudiar y tenerlos en consideración atendiendo a su firme decisión y deseo de estudiar. 


\section{Barreras vocacionales y bajo rendimiento}

Un cuarto grupo de razones encontradas en el análisis del discurso de once de los entrevistados que iniciaron sus estudios, alude a barreras vocacionales y a deserción por bajo rendimiento académico. En el modelo teórico presentado (Tinto 1975) la falta de integración al sistema académico medida por la motivación por el estudio, la satisfacción con la especialidad elegida y el rendimiento académico constituye uno de los factores centrales asociados a la deserción temprana.

Revisaremos en primer lugar los testimonios referidos a las barreras vocacionales, las opciones limitadas y la falta de información. Como se aprecia en los siguientes testimonios, encontramos falta de información sobre el contenido y exigencias de las carreras elegidas, opciones limitadas por la oferta de cada IES, así como motivaciones insuficientes, tanto en el caso de la informante que decide ingresar «a ver qué pasa», como en el de aquellos que claramente tienen otra prioridad vocacional:

Yo quería la carrera de ingeniería ambiental, pero estaba en ingeniería civil, y el coordinador académico no quiso que me cambie, así que me salí antes de perder más el tiempo (FI4).

Yo le digo [a mi mamá], la única universidad que ha llegado hasta aquí es la Católica y hay [ingeniería] agroindustrial y ambiental, y me dice ipero a ti no te gustan esas carreras! Y le digo pero voy a postular a ver cómo me sale, pero no me daba ilusión porque no me gustaban las carreras. Me puse mal, me dio como depresión. Estuve internada en la posta de Canchaque [al regresar a su pueblo] por una semana, y mi mamá decidió quitarme de la beca, y ya pues (FI9).

[Ingresé) a ingeniería civil [en Tacna] porque me gustaban los números. La que me gustaba era contabilidad. Tenía temor, es que como no era mi carrera sino que era lo que había. Me decían: si no es tu carrera no te va a ir bien, y ya pues yo también tenía esa duda. Yo tenía temor pues me dijeron que si yo me retiraba cuando ya estaba estudiando tenía que devolver [el dinero], hasta que al final les dije no, no voy a seguir con la beca (FI12).

Además de sentirme sola [quería que su papá la acompañe], averigüé más sobre la carrera que había escogido y no me gustó. La carrera no es lo que había pensado. Antes de elegir una carrera deberían pensar bien en lo que van a hacer (FI33).

[Dejar Beca 18] fue decisión mía, como te digo a mí me encanta la música (es arpista en Huanta) y como estar en el taller no me ayudaba con la música, mis padres me dijeron; ya, es decisión tuya, y así lo hice. Si me hubieran dado la 
posibilidad de una carrera de música o en la que pueda seguir con la música si hubiera seguido (MI5).

En cuanto a problemas de rendimiento académico e insuficiente orientación y tutoría presentamos los testimonios siguientes:

El curso era bastante nuevo para mí, costó un poquito aprender. Faltó acompañamiento, tutoría también, no teníamos quien nos oriente. Los libros costaban caro y no teníamos el dinero para conseguirlos. Como vivía solo, había que cocinar, lavar, madrugar para el desayuno, eso era medio dificultoso para estudiar. [Desaprobó un curso], eso fue lo que influyó en salir de la beca (MI26).

El nivel académico en Lima es mucho más que acá en Huancavelica y yo no iba a estar lo suficientemente capacitada para seguir la carrera de ingeniería civil. El docente que me atendió me dijo que llegando a Lima iba a ir a una academia por unos meses y de ahí iba a entrar de frente a la universidad. Dije no, yo pensaba postergar la beca un año y en ese año prepararme bien. El docente dijo no, no puedes postergar, si no renuncia, me dijo (FI22).

[Al inicio de los estudios] sufrí bastantes cambios, era bien distinto, un poco más difícil, en parte la verdad en algunos de los cursos como que no estaba muy bien. Desde el inicio lo anunciaban [al tutor] que ya se va a asignar, ya se te va a asignar y nada (MI24).

[Lo más difícil] era la exigencia académica, te exigen bastante, pero hay que esforzarse, ¿no? El primer ciclo me chocó bastante y jalé [dos de siete cursos] y de ahi perdí la beca. No hay tutor en el primer ciclo en la universidad, recién en el segundo ciclo, cuando yo ya no estaba (FI20).

Como se aprecia en los testimonios anteriores, el bajo rendimiento académico (o el temor al mismo) se asocia a la orientación y tutoría insuficientes durante el primer ciclo de estudios. Se conoce que estos ciclos iniciales son claves para la adaptación académica y social al nuevo contexto de vida. Estos factores están además asociados a problemas afectivos por la lejanía de sus hogares y a dificultades de adaptación y exigencias del nuevo tipo de vida como estudiantes viviendo solos en un ambiente que les es ajeno y desafiante.

\section{Dificultades en el proceso de postulación y cumplimiento de condiciones}

Finalmente presentamos los testimonios referidos a problemas en el proceso de postulación y aceptación de Beca 18. Encontramos este tipo de razones en el 
discurso de diez de los entrevistados; sin embargo esta no constituye la razón principal de deserción. Aparece más bien en combinación con otras razones.

Lo primero que apreciamos en los siguientes testimonios es la falta de información o información errada sobre condiciones y requisitos del programa y sobre las exigencias de los estudios:

Algunas dudas ya tuvieron [sus padres] porque decian que cuando terminabas de estudiar el Estado se quedaba con tu sueldo. Así hablaban en mi pueblo (MI23).

Escuché rumores que no se les depositaba mensualmente a los estudiantes que se iban fuera de Huancavelica. Eso también influyó fuertemente [además de su temor de no contar con el nivel académico suficiente] en mi decisión de no aceptar la beca, porque si no me depositaban mensualmente yo qué iba a hacer, porque mis padres no cuentan con las posibilidades económicas para que me puedan estar mandando dinero (FI22).

Pero yo tenía temor, pues me dijeron que si yo me retiraba cuando ya estaba estudiando tenía que devolver, me iban a botar de la beca [tenía temor a fracasar en los estudios de una carrera que no le gustaba] y encima tenía que devolver todo lo que me habían dado. Yo también me había asustado encima va a ser más gastos para mis padres. Dije no, mejor me salgo, dije (FI12).

Tengo amigos de acá estudiando en Lima también por Beca 18 y me cuentan sus experiencias. Es demasiado ajetreada la vida en la universidad privada (UPCH) y estudian hasta sábado y si es posible domingo y prácticamente no tienen días libres. Entran desde las siete u ocho y tienen clases hasta las cinco, no les dan refrigerio nada, ni les permiten comer en horas de clase ni una fruta para que se alimenten, nada (FI13).

Estos relatos permiten apreciar que los rumores pueden tergiversar los requerimientos reales del programa, en especial en lo referido a las condiciones económicas que también aparecen en las barreras económicas ya descritas. Es asimismo evidente que falta información sobre las condiciones de estudio y de vida para los becarios de provincias que eligen estudiar en Lima.

Otro tema señalado por los entrevistados como factor que influye en la deserción es el de las dificultades del proceso de postulación, los trámites complicados y el incumplimiento de plazos:

[El proceso de postulación] fue difícil porque nos hacían que llenáramos papeles, todo pues llenamos... como no sabíamos manejar computadora teníamos que subir y pagar para que lo hagan (IM28). 
El trámite era demasiado, mucho ya, cualquier error... Todo era internet, como había salido del colegio no tenía mucha experiencia, tuve dificultades en eso (MI11).

No hay que imponer muchas trabas, es suficiente con dar a conocer lo que realmente nosotros gastamos. No sé si lo hacen por lo que vale la contabilidad, pero creo que estresaba un poco cuando teníamos examen y teníamos que presentar papeles, [confeccionar] el horario, presentar las facturas... como que mucho, se te amontonaban y ya, pues (FI9).

[En el proceso de postulación] sí hubo problemas porque a mi provincia el listado de los ingresantes llegó tarde y al día siguiente me dijeron que tenía que presentarme en la UPC (MI17).

[Recomiendo al programa] que cumplan con los plazos para el pago del dinero y también para la respuesta sobre los exámenes, a veces decían que el 21 de febrero van a dar [los resultados], no, es para el 21 de marzo, no, es para fines de abril y así nos tenían... ya tanto así que tuve que renunciar (FI18).

En parte sinceramente [la culpa] fue mía... Yo debí quedarme [en Lima] y ni siquiera aparecerme por aquí (Moquegua), en realidad no había terminado ni siquiera el ciclo, y como era 28 de julio y tenías unos tres o cuatro días ahí... un fin de semana largo, y yo decidí venir... Solo faltaba alguno que otro [examen] ... extrañaba [y no regresó a tiempo y perdió la matrícula para el segundo ciclo) (MI24).

Las dificultades procesales incluyen dificultades para el llenado virtual de los formularios, pues estos jóvenes rurales no están familiarizados con el uso de páginas web, la exigencia de documentar y reportar gastos, horarios y trámites, incluso en épocas de exámenes, así como demoras en recibir los resultados de los exámenes (para los ingresantes) y en el pago de sus asignaciones mensuales (para los que están estudiando). Es posible, sin embargo, que las dificultades procesales señaladas oculten un problema de bajo rendimiento, pues este es el caso de varios de los entrevistados que aducen estas razones. Pese a ello, es obvio que se necesita simplificar los trámites, revisar las exigencias de rendición de cuentas y cumplir con los plazos de notificaciones y entrega del dinero, como precisamos en la sección final de este trabajo.

\section{El futuro, hoy}

En esta sección final del análisis nos referimos a las actividades que hoy desempeñan los jóvenes entrevistados que, como se ha señalado, desertaron o se 
apartaron del programa por diversas razones. Lo primero que cabe resaltar es que la gran mayoría desea seguir estudiando, y que 17 de los 32 que contestaron a la pregunta sobre su actividad actual están siguiendo las carreras técnicas o profesionales de su preferencia sin contar con la ayuda de Beca 18.

Continúo con ingeniería de minas porque, como se dice, hay que seguir para adelante, no hay que retroceder para atrás (MI26).

Justamente ahora estoy comenzando el segundo semestre, tengo que terminar la carrera solventándome por mí mismo y así normal (MI17).

Sin embargo, no en todos los casos es factible estudiar la carrera elegida, ya sea por su costo o por no estar disponible en los IES cercanos a su hogar. Pese a ello, lo que en realidad importa, según los testimonios, es alcanzar la educación superior:

Tenía que estudiar una carrera, ni modo que me iba a quedar sin estudiar. Tenía que haber otras salidas (FI25).

La segunda evidencia es que la mayoría, en especial las mujeres, están estudiando en la localidad de su hogar de origen o en un centro cercano a él. Ello les ha permitido obviar las barreras afectivas y de adaptación a un medio extraño.

[Estoy estudiando] en Puquio en un instituto. [P. ¿ Y cómo así te fuiste hasta Puquio?]. Porque mi hermana vivía aquí y me llamaron (MI23).

Sin embargo, una tercera evidencia es que las barreras económicas aparecen como fuertes limitantes: quince jóvenes, en su mayoría varones, no han podido seguir estudiando o estudian y trabajan en forma eventual o con sus familias. En algunos casos su situación es dramática:

Hago todos mis esfuerzos, trabajo, estudio. Trabajo en una panadería de tres a cinco de la mañana, de ahí siete de la mañana voy a estudiar, de ahí a las doce voy a un restaurante hasta las cuatro, de ahí hago mis tareas hasta las siete, de ahí me voy a otro sitio, a un chifa (MI30).

Yo quisiera estudiar en la SENATI, pero la pensión es muy cara y no creo que lo logre (MI32).

Estoy trabajando, quería estudiar una carrera corta, pero [tengo que] trabajar y juntar para estudiar (FI18).

Es también notable el compromiso de la mayoría de estos jóvenes por salir adelante y servir de apoyo a sus familias: 
Bueno me gustaría terminar los tres años que tengo que estudiar, trabajar, apoyar a mi familia y, bueno, trabajar y ganar dinero y apoyar a mi mamá porque es la que necesita, mis hermanos también son cuatro y falta tiempo para que terminen y, como mis papás son mayores, entonces ya pues, la que más puede apoyar soy yo (FI33).

Concluyendo, estos testimonios revelan que la gran mayoría de estos jóvenes tiene una alta motivación para seguir estudios terciarios y que, en efecto, muchos han podido continuar haciéndolo. Sin embargo se aprecia asimismo que han tenido que optar por IES de menor calidad, pero que están más cerca de sus hogares y tienen costos menores. Muchos de los que no cuentan con el apoyo económico de sus padres, han postergado su ingreso a una institución de educación superior, o deben trabajar y estudiar al mismo tiempo. Por ello todos los esfuerzos que pueda realizar Beca 18 para disminuir la deserción, que ya es bastante baja, redundará en beneficio de estos jóvenes que sin duda merecen la mayor atención de parte del Estado.

\section{CONCLUSIONES Y SUGERENCIAS}

A pesar de que este estudio se centró en un programa específico, Beca 18, pensamos que las conclusiones son extrapolables a otros programas de becas en la región. Otros países culturalmente diversos con grandes brechas socioculturales como el nuestro enfrentan situaciones parecidas, por lo que la reflexión que la realidad de Beca 18 ha motivado en el presente estudio puede ser de utilidad cuando se enfrenten retos similares. Con base a los testimonios presentados y al análisis de la información documental y base de datos de Beca 18, seguidamente proponemos algunas conclusiones y sugerencias que consideramos pueden incidir en disminuir la deserción temprana y mejorar la calidad y eficacia de los programas de becas para jóvenes de bajos recursos en contextos de alta diversidad cultural y social.

a) No existen diferencias significativas entre continuadores y desertores en lo referido a características socioeconómicas individuales y familiares. El enfoque cuantitativo, utilizando un modelo Probit, esperaba encontrar diferencias entre estos dos grupos en cuanto a edad, sexo, tipo de institución educativa (privada vs. pública) y el nivel de pobreza de la familia de origen. Con el modelo ajustado, encontramos que ninguna de las variables analizadas genera un cambio mayor de $1 \%$ sobre la probabilidad de desertar o no a la 
beca. De esa manera, se constata que las variables cuantitativas no brindan evidencia concluyente sobre las diferencias entre los becarios desertores y los continuadores. En otras palabras, a pesar de ser las más significativas, ni las características individuales (mayor edad), la mayor precariedad económica (tipo de alumbrado del hogar) ni las características de los padres (menor educación y trabajo de la madre) tienen la suficiente relevancia, en términos estadísticos, para explicar las diferencias entre los desertores y los continuadores. Por ello nos centramos en las dimensiones cualitativas (contexto familiar y afectivo) para entender las razones de la renuncia a la beca o de la deserción temprana.

b) Apoyo emocional en la inserción al nuevo contexto. Como se ha señalado, las dimensiones afectivas (lejanía de la familia, miedo al nuevo contexto, etc.) aparecen en los testimonios como la causa principal de la no aceptación de la beca o de la deserción temprana. Para disminuir la incertidumbre, el temor y la sensación de aislamiento en el período de aceptación y el inicio de los estudios, se sugiere crear lazos sociales entre los becarios procedentes de una misma región o que van a una misma IES. Ello se podría lograr creando comunidades virtuales una vez realizada la selección para poner en contacto por vía virtual a los becarios. Pese a que existe una red virtual de becarios, denominada «intranet del becario», esta se activa solo una vez que inician sus estudios. En las etapas previas no se ha previsto un mecanismo de articulación y socialización entre los seleccionados. Por ello es necesario que los sistemas de gestión virtual, como la intranet, no sean solo medios administrativos, sino que también puedan permitir a los jóvenes acceder a foros de ayuda y guías interactivas desde que son seleccionados y de esa forma acompañarlos desde el inicio del proceso como parte de la tutoría que las IES deben brindarles.

Otra medida clave es lograr que los IES participantes en el programa presten apoyo en la selección de los lugares de residencia para los becarios, en especial los que viajan a Lima o a ciudades grandes. Las universidades peruanas en general, y las privadas en especial, no cuentan con residencias estudiantiles, pero, como se hace en otros países, pueden proporcionar a los becarios listas de residencias particulares que cuenten con garantía de seguridad, condiciones adecuadas de habitabilidad, accesibilidad al IES y precios razonables. Ello disminuiría la sensación de desamparo e incertidumbre que los padres y becarios, en especial las jóvenes, sienten al tener que buscar ellos mismos un lugar donde vivir en la ciudad de destino. 
El apoyo sicológico al becario durante el ciclo inicial de estudios es asimismo clave para la orientación del estudiante que enfrenta un ambiente desconocido y culturalmente diferente del de origen. Varias de las IES que participan en Beca 18 ya cuentan con mecanismos de apoyo emocional y social al becario. Por ejemplo, el «amigo PUCP» que recluta voluntarios entre estudiantes de la institución para el acompañamiento e inserción social del becario. Esta práctica debe evaluarse y reforzarse en todos los IES participantes.

Finalmente, en el proceso de entrevistas y selección de becarios, especialmente para los de mayor edad, es necesario indagar y evaluar si la presencia de vínculos afectivos con sus parejas (enamorados, novios o «compromisos») pueda constituir un obstáculo para la aceptación de la misma y para emprender los estudios. Obviamente ello debe hacerse con el respeto y cuidado que el tema requiere.

c) Información y apoyo económico oportuno en las fases iniciales del proceso. Como se ha demostrado, muchos jóvenes no cuentan con los recursos mínimos para solventar los gastos que la postulación y la fase inicial de los estudios requieren. Una primera medida es mejorar la puntualidad de los pagos para solventar los gastos de alojamiento, comida, transporte y otros en las primeras semanas de estudios. Sería útil asimismo proporcionar información sobre los costos reales de instalación y mantenimiento en el lugar de destino para evitar la desinformación y la inseguridad y temor que ello conlleva. Es importante asimismo notificar a los seleccionados con anticipación sobre las fechas en que deben viajar y los trámites que deben solventar para que, en caso que el programa no pueda adelantarles los fondos, puedan conseguir préstamos para cubrir estos gastos. Sería también pertinente considerar la simplificación de los documentos y trámites exigidos para que estos no constituyan una barrera de costos y tiempo innecesaria para los jóvenes.

d) Apoyo en los casos de enfermedad. Entre los 34 entrevistados se encontraron siete casos en los que los problemas de salud, propios o de familiares, constituyeron parte de las razones para renunciar a Beca 18. Queremos hacer hincapié en los casos en los que los problemas de salud del becario son la única razón para no poder iniciar los estudios, en especial en aquellos casos en que es evidente una enorme motivación por no perder la beca y confiar que luego de su recuperación, puedan volver a acceder a ella. En estos casos los becarios se han acercado al programa para explicar su situación, insistiendo 
en que no se trata de un retiro voluntario. Pese a que se evidencia voluntad de los funcionarios en atender cada caso, ninguno de los entrevistados que ha sufrido problemas de salud ha recibido respuesta positiva hasta el momento de culminar este estudio. Sugerimos que en estos casos se permita a estos jóvenes postergar el inicio de la beca, pues no solo se están sobreponiendo a duras enfermedades, sino que, de no atenderse su reclamo, perderían la oportunidad de estudiar. Se ha encontrado que los jóvenes consideran que las dolencias menores o enfermedades de familiares les dan derecho de postergar el inicio de los estudios, razón por la cual Beca 18 debe informar claramente que no es posible esta postergación para evitar malentendidos.

e) Mejorar la información y la orientación vocacional. Otra de las razones encontradas para la deserción, especialmente entre los que iniciaron sus estudios, es la falta de información sobre la carrera elegida, así como también limitadas opciones para estudiar la carrera que verdaderamente les interesaba. Es evidente que la política de Beca 18 es priorizar aquellas carreras en las que existe un déficit de especialistas en el país, pero esto debe revisarse periódicamente. Más precisamente, es clave reforzar la orientación vocacional para los becarios al momento de elegir la carrera. Muchos de estos eligen carreras sin conocer bien lo que ellas suponen, su campo de aplicación, las competencias requeridas, etc. En este tema los entrevistados coinciden en señalar la ausencia de tutorías, tanto para la orientación vocacional como para mejorar su rendimiento académico, estas se inician muy tarde cuando la etapa crítica es el ciclo inicial, incluyendo el ciclo 0 .

Mejorar la eficiencia de Beca 18 pasa por proporcionar a los postulantes información más detallada y precisa del abanico de carreras y especialidades ofrecidas. Ello se puede lograr no solo con consejerías individuales sino también poniendo a disposición de los postulantes información virtual sobre cada carrera proporcionada por las IES participantes en el programa. De ser factible, se puede vincular a los interesados en una carrera particular con los mejores estudiantes de cada especialidad que quieran colaborar en orientar a los becarios en los detalles de la misma por vía virtual. Ello no tiene un costo alto y puede evitar una mala selección y una renuncia a la beca cuando ya es demasiado tarde para cambiar de decisión sobre la carrera elegida.

f) Mejoras en la información sobre el proceso de postulación y requisitos de los estudios. Finalmente, se ha encontrado que muchos jóvenes que desertaron 
lo hicieron por contar con información errada sobre las condiciones y requisitos de Beca 18, en especial en lo referente a condiciones económicas. Es evidente que corren rumores que alimentan esta desinformación, como los referidos a que luego de terminar sus estudios deberán trabajar gratis para el Estado. También es cierto que alguna de esta información es real y revela fallas en el sistema, como por ejemplo la referida a demoras en los pagos y costos asociados a la reprobación de cursos o retiro de ellos. Es asimismo aconsejable que las IES participantes complementen el acceso a sus web con un sistema de consulta (vía línea gratuita o «chat»), para que los becarios puedan obtener mayor información sobre los estudios, fechas claves, la vida universitaria, horarios, facilidades, requisitos académicos, etc.

Además, es aconsejable simplificar los trámites y documentos solicitados atendiendo al tema de tiempo y costos para estos jóvenes, que como hemos señalado, tienen serias dificultades para solventar incluso gastos mínimos asociados al proceso de postulación e inicio de los estudios. También consideramos recomendable que los encargados de las Unidades de Enlace Regional de las distintas regiones y provincias tengan un conocimiento preciso sobre los procedimientos, de modo que no ocurran desinformaciones, malentendidos ni problemas con los interesados, beneficiarios y becarios. Para ello es necesario contar con protocolos de atención a los jóvenes para cada convocatoria y que los encargados de las regiones sean capacitados periódicamente.

A modo de balance, puede señalarse que Beca 18 es un programa altamente valorado por estos jóvenes, pese a ser desertores, y que los temas pendientes son ajustes finos a la operatividad del programa que permitirán hacerlo más inclusivo, equitativo y relevante. Esperamos haber contribuido en cierta medida a ello con este breve estudio.

\section{REFERENCIAS}

Balmori, Elsa, María Teresa de la Garza, Elda Reyes (2010). El modelo de deserción de Tinto como base para la planeación institucional: el caso de dos instituciones de educación superior tecnológicas. Ponencia $\mathrm{N}^{\circ} 16$ en el XI Congreso Nacional de Investigación Educativa, México.

Casillas, Miguel, Adrián De Garay, Julia Vergara y Mónica Puebla (2001). Los estudiantes de la UAM-A, un sujeto social complejo. Revista Mexicana de Investigación Educativa, 6(11), 139-163. 
Casillas, Miguel, Ragueb Chaín y Nancy Jácome (2007). Origen social de los estudiantes y trayectorias estudiantiles en la Universidad Veracruzana. Revista de la Educación Superior, XXXVI (2), 142, 7-29.

Cinda (2006). Repitencia y deserción universitaria en América Latina. Santiago: Cinda, IELSAC y Universidad de Talca. Recuperado de http://www.cinda.cl/download/ libros/Repitencia\%20y\%20Deserci\%C3\%B3n\%20Universitaria\%20en \%20 Am\%C3\%A9rica\%20Latina.pdf

Cueto, Santiago, Alejandra Miranda y Maria Crsitina Vásquez (2016). Inequidades en educación. En GRADE Investigación para el Desarrollo en el Perú: once balances. Lima: GRADE.

Instituto Nacional de Estadística e Informática - INEI (2010). Censo Nacional Universitario: Base de datos REDATAM en: http//censos.inei.gob.pe/censos/redatam_inei/

Instituto Nacional de Estadística e Informática - INEI (2010). Encuesta Nacional de Hogares 2010: http://www.inei.gob.pe/biblioteca.virtual/publicaciones.

Lavado, Pablo y José Gallegos (2005). La dinámica de la deserción escolar en el Perú: un enfoque usando modelos de duración. Informe Final de Proyecto CIES: Lima. Recuperado de http://repositorio.minedu.gob.pe/bitstream/ handle/123456789/253/128.\%20La\%20din\%C3\%A1mica\%20de\%20la\%20 deserci\%C3\%B3n\%20escolar\%20en\%20el\%20Per\%C3\%BA\%20Un\%20enfoque $\% 20$ usando $\% 20$ modelos $\% 20$ de $\% 20$ duraci $\%$ C3\%B3n.\%20Informe $\% 20$ final $\% 20 \mathrm{de} \% 20$ proyecto.pdf?sequence=1\&isAllowed=y, pp. 13-15.

McLauchlan, Patricia y Arturo Miranda (2000). Poverty and Education in Perú. En Fernando Reimers (ed.), Unequal schools, unequal chances: The challenges to unequal opportunities in the Americas (pp. 376-399). Cambridge: Harvard University Press.

Miller, Dinorah (2009). La equidad en la universidad. El Programa Nacional de Becas (Pronabes) y la condición de la juventud de los estudiantes. Una mirada desde la UNAM. México: ANUIES.

Núñez Cuba, Diego (2018). Transitando de la Vocación a la Profesión: El caso de los estudiantes de Beca Vocación Maestro en la PUCP. Tesis de licenciatura en Sociología. Lima: Facultad de Ciencias Sociales, PUCP.

Silva, Marisol (2011). El primer año universitario. Un tramo crítico para el éxito académico. Perfiles educativos, 33, 107-111. Recuperado de http://www.scielo.org. $\mathrm{mx} /$ scielo.php?script=sci_arttext\&pid=S0185-26982011000500010,

Tinto, Vincent (1975). Dropout from higher education: A theoretical synthesis of recent re-search. Review of Educational Research, 45, 89-125. https://doi. org/10.2307/1170024 
Tinto, Vincent (1993). Leaving College: Rethinking the causes and cures of student attrition. 2nd ed. Chicago: University of Chicago Press. https://doi.org/10.7208/ chicago/9780226922461.001.0001

Torres, Luz (2012). Retención estudiantil en la educación superior: revisión de la literatura y elementos de un modelo para el contexto colombiano. Bogotá: Editorial Pontificia Universidad Javeriana. Recuperado de http://www.javeriana.edu.co/ documents/15838/273636/Retenci\%25C3\%25B3nEstudiantil22.pdf/124fdba52318-432a-8e9f-126a2501c229, pp. 27-28, 51-55.

Voigt Lydia y Jim Hundrieser (2008). Student Success, Retention, and Graduation: Definitions, Theories, Practices, Patterns, and Trends. Noel-Levitz Retention Codifications. Recuperado de http://www.stetson.edu/law/conferences/highered/ archive/media/Student $\% 20$ Success, $\% 20$ Retention,\%20and\%20Graduation-\%20 Definitions,\%20Theories,\%20Practices,\%20Patterns,\%20and\%20Trends.pdf, pp. 6-8. 